\title{
Downregulation of IRS-1 promotes metastasis of head and neck squamous cell carcinoma
}

\author{
XUAN LUO ${ }^{1 *}$, SONGQING FAN $^{2 *}$, WENBIN HUANG $^{3}$, SULAN ZHAI $^{1}$, ZHUO MA $^{1}$, PING LI $^{1}$, \\ SHI-YONG SUN ${ }^{4}$ and XUERONG WANG ${ }^{1}$ \\ ${ }^{1}$ Department of Pharmacology, Nanjing Medical University, Nanjing, Jiangsu; ${ }^{2}$ Department of Pathology, \\ The Second Xiang-Ya Hospital, Central South University, Changsha, Hunan; ${ }^{3}$ Department of Pathology, \\ Nanjing Medical University Affiliated Nanjing First Hospital, Nanjing, Jiangsu, P.R. China; ${ }^{4}$ Department of Hematology \\ and Medical Oncology, Winship Cancer Institute, Emory University School of Medicine, Atlanta, GA, USA
}

Received February 23, 2012; Accepted May 4, 2012

DOI: $10.3892 /$ or.2012.1846

\begin{abstract}
Lymph node metastasis is responsible for the high morbidity of head and neck squamous cell carcinoma (HNSCC). To date, the role of insulin receptor substrate 1 (IRS-1) in tumorigenesis and metastasis of head and neck cancer has not been elucidated. In this study, we found a negative correlation of IRS-1 expression with tumor metastasis both in human tissue samples and in cell lines. Furthermore, we found that knockdown of IRS-1 expression enhanced cell invasive potency and induced EMT in parallel with upregulation of miR-9 expression. We propose that IRS-1 suppresses metastasis of head and neck cancer possibly through miR-9.
\end{abstract}

\section{Introduction}

Head and neck cancer is the eighth most common cancer and accounts for $3 \%$ of all cancers in men in the United States. The estimated new cases for both male and female are $\sim 36,540$ and expected deaths are 7,880 in 2010 (1). Lymph node metastasis of head and neck squamous cell carcinoma (HNSCC) is a strong indicator of prognosis with a 5-year survival rate of $<50 \%$ for patients with unilateral lymph node metastasis and a survival rate of $<25 \%$ for patients with bilateral metastasis. Despite the advances in combining chemotherapy with surgery and radiotherapy, survival rates for patients with recurrent head and neck cancer remain poor $(2,3)$. Therefore, identifying the crucial proteins and clarifying mechanisms that contribute

Correspondence to: Professor Xuerong Wang, Department of Pharmacology, Nanjing Medical University, 140 Hanzhong Road, Nanjing, Jiangsu, P.R. China

E-mail: wangxrwn@hotmail.com

*Contributed equally

Key words: insulin receptor substrate 1, head and neck squamous cell carcinoma, metastasis, E-cadherin, miR-9 to metastasis will be significant to provide biomarkers for prognosis and targets for treatment.

Insulin receptor substrate 1 (IRS-1) is an adaptor protein that integrates multiple transmembrane signals from growth factors and hormones, to regulate cell growth, survival, differentiation and metabolism (4). Previous studies mainly focused on its roles in metabolism, but recently it was reported to be correlated with cancer progression and metastasis (5). As a major substrate of insulin and insulin-like growth factor I (IGF-I) and mainly functioning through activating PI3K/Akt and MAPK pathway, IRS-1 has been considered oncogenic in cancer progression. As well, elevated expression levels and the active form of IRS-1 has been reported in a variety of tumors, including hepatocellular carcinomas (HCCs), pancreatic cancer, breast cancers, leiomyomas, Wilms' tumors, rhabdomyosarcomas, liposarcomas, leiomyosarcomas and adrenal cortical carcinomas $(5,6)$. However, recently Houghton et al (7) showed increased cell proliferation after silencing of IRS1 gene expression in A549 cells suggesting tumor suppression potential. Furthermore studies in tumor metastasis are quite limited and controversial. IRS-1 has been identified as both a positive and negative regulator of metastasis in breast cancer (8-10). While in lung cancer, IRS-1 has been confirmed to suppress tumor metastasis (11). To date, its role in head and neck cancer has not been identified.

The epithelial-mesenchymal transition (EMT) plays important roles in tumor metastasis. Cells undergoing EMT lose their epithelial characteristics and phenotype, acquire mesenchymal properties, and thus gain enhanced motility (12). EMT is accompanied by decreased E-cadherin expression and increased vimentin expression. Many transcriptional factors such as snail, slug, twist and bmi1 have been confirmed to suppress E-cadherin transcription and thus identified as both molecular markers and inducers of EMT $(13,14)$. Loss of E-cadherin, not only results in loss of adherens junctions between neighbor cells resulting in dissemination of cells from the original tumor, but also results in releasing its cytoplasmic binding with $\beta$-catenin. $\beta$-catenin, when translocating to and accumulating in the nucleus, induces transcription of other oncogenes involved in tumor progression, malignancy and metastasis (15). Therefore, loss of E-cadherin 
expression is not only the initial and essential step of EMT, but also a potential promoter of EMT.

MicroRNAs (miRNAs) are 22 -nt non-coding RNAs that negatively regulate gene expression at the post-transciptional level via direct binding with the 3 ' untranslated regions (UTR) of target mRNAs $(16,17)$. Many miRNAs have been implicated to play important roles in tumor metastasis, such as miR-21, miR-31, miR-125a, miR-155 and miR-373 (18,19). Recently, miR-9 has been shown to directly target E-cadherin to reduce its expression at post-transcription levels and thus induce EMT (20). miR-200 family members and miR-10b have been confirmed to indirectly regulate E-cadherin expression and induce EMT $(21,22)$.

In this study, we investigated the role of IRS-1 in head and neck cancer metastasis. First, we found the expression of IRS-1 protein in head and neck squamous cell carcinoma (HNSCC) with lymph node metastasis decreased compared to HNSCCs without metastasis. Moreover, we found IRS-1 mRNA expression in metastatic lymph node tumor tissues to be decreased compared to primary tumor tissues of HNSCCs. In a paired poorly-highly metastatic HNSCC cell model, we identified decreased expression of IRS-1 in highly metastatic cells. We then showed that the invasive potency of poorly metastatic cells (which express high levels of IRS-1) increased after downregulation of IRS-1 expression. In addition, we found downregulation of E-cadherin and upregulation of miR-9 after IRS-1 knockdown. These results suggest that decreased expression of IRS-1 is correlated with HNSCC metastasis and reduction of IRS-1 promotes invasion and EMT of HNSCC cells possibly through upregulation of miR-9.

\section{Materials and methods}

Human tissue samples. In this study, we used two cohorts of human tissue samples from different cities of China. One cohort contained formalin-fixed paraffin-embedded (FFPE) tumor tissue sections collected from the Department of Oral Surgery and Otolaryngology-Head and Neck Surgery at the Second XiangYa Hospital of Central South University (Changsha, China) from 2006 to 2010. Of the 108 HNSCC patients, 84 were males and 24 were females (mean age, 56.5 years). The HNSCC histological patterns and clinical $\mathrm{T}$ stages were determined according to WHO histological classification of the HNSCC and the TNM classification of malignant tumors (Table I). Of the patients included in the study, 33 had local lymph node metastasis and 75 had negative lymph nodes. No case revealed distant metastasis, so that all of the cases were classified as M0. These specimens were subjected to immunohistochemical (IHC) analysis.

The other cohort contained FFPE tumor tissues collected from paired primary tumor and metastatic lymph node of 10 HNSCC patients at Nanjing Medical University Affiliated Nanjing First Hospital (Nanjing, P.R. China) from 2007 to 2011. Of the 10 patients, 7 were males and 3 were females (mean age, 71.2 years). These samples were subjected to RNA extraction and subsequent quantitative RT-PCR analysis of IRS-1 expression.

Reagents. Rabbit polyclonal anti-insulin receptor substrate 1 antibody was purchased from Upstate Group, Inc. (Charlottesville,
Table I. Analysis of the association between IRS-1 protein expression and HNSCC.

\begin{tabular}{|c|c|c|}
\hline \multirow[b]{2}{*}{ Clinicopathological features } & \multicolumn{2}{|c|}{ IRS-1 } \\
\hline & Positive $(\%)$ & Negative $(\%)$ \\
\hline \multicolumn{3}{|l|}{ Age } \\
\hline$\leq 50$ years $(n=43)$ & $31(72.1)$ & $12(27.9)$ \\
\hline$>50$ years $(n=65)$ & $45(69.2)$ & $20(30.8)$ \\
\hline \multicolumn{3}{|l|}{ Gender } \\
\hline Male $(n=84)$ & $60(71.4)$ & $24(28.6)$ \\
\hline Female $(n=24)$ & $16(66.7)$ & $8(33.3)$ \\
\hline \multicolumn{3}{|l|}{ Histological grade } \\
\hline $\mathrm{I}(\mathrm{n}=18)$ & $16(88.9)^{\mathrm{a}}$ & $2(11.1)$ \\
\hline II $(\mathrm{n}=70)$ & $51(72.9)^{\mathrm{b}}$ & $19(27.1)$ \\
\hline III $(n=20)$ & $9(45.0)$ & $11(55.0)$ \\
\hline \multicolumn{3}{|l|}{ Clinical stage } \\
\hline$I(n=36)$ & $31(86.1)^{c}$ & $5(13.9)$ \\
\hline II $(n=39)$ & $31(79.5)^{\mathrm{d}}$ & $8(21.5)$ \\
\hline III $(n=33)$ & $14(42.4)$ & $16(57.6)$ \\
\hline \multicolumn{3}{|l|}{ Lymph node status } \\
\hline LNM (n=33) & $22(66.7)$ & $11(33.3)$ \\
\hline No LNM (n=75) & $54(72.0)$ & $21(28.0)$ \\
\hline
\end{tabular}

Chi-square test: ${ }^{\mathrm{a}}$ grade I vs. grade III, $\mathrm{p}<0.01$, ${ }^{\mathrm{g}}$ grade II vs. grade III, $\mathrm{p}<0.05$; ${ }^{\mathrm{c}}$ stage I vs. stage III, $\mathrm{p}<0.01$, ${ }^{\mathrm{d}}$ stage II vs. stage III, $\mathrm{p}<0.01$. LNM: lymph node metastasis.

VA). Mouse monoclonal anti-E-cadherin and anti-vimentin antibodies were purchased from Santa Cruz Biotechnology, Inc. (Santa Cruz, CA). Mouse monoclonal anti-tubulin antibody was purchased from Sigma Chemical, Co. (St. Louis, MO). Sulforhodamine B was purchased from Sigma Chemical, Co., dissolved in $1 \%(\mathrm{v} / \mathrm{v})$ acetic acid at a concentration of $0.4 \%(\mathrm{w} / \mathrm{v})$ and stored at room temperature.

Cell lines. Paired low and high metastatic HNSCC cells, 686LN and 686LN-M4e, have been described (23-25). Briefly, 686LN cell line was derived from lymph node metastasis of a basal-tongue primary tumor. It has low capability for metastasis to lymph node and lung when injected into the mylohyoid muscle near the FOM (floor-of-mouth) of nude mice. When the primary tumor reached a length of $2.0 \mathrm{~cm}$, the cervical lymph nodes were collected. 686LN cell lines derived from the metastatic lymph nodes were cultured in vitro, then re-injected to another mouse as described above for further selection. After four rounds of in vivo selection, the highly metastatic 686LNM4e cell line was established, which showed 100\% lymph node metastasis and $90 \%$ lung metastasis in nude mice in vivo. 686LN-M4e also showed much higher Matrigel invasion capability than the parental cells (23-25). These cell lines were grown in monolayer cultured in Dulbecco's modified Eagle's medium (DMEM)/F12 medium supplemented with 5\% fetal bovine serum at $37^{\circ} \mathrm{C}$ in a humidified atmosphere consisting of $5 \% \mathrm{CO}_{2}$ and $95 \%$ air. 
Gene knockdown by small interfering RNA. IRS1 SMARTpool siRNA reagent, which contains four different targeting sequences and correlated control siRNA were purchased from Upstate Group, Inc. Cells were seeded to 6-well plates at $5 \times 10^{5}$ cells/well and transfected with $100 \mathrm{nmol} / \mathrm{l}$ siRNA for $24 \mathrm{~h}$ using Lipofectamine 2000 Transfection Reagent from Invitrogen Life Technologies (Carlsbad, CA). After transfection for $24 \mathrm{~h}$, cells were reseeded and subjected to an invasion assay or reseeded to 96 -well plates for subsequent Sulforhodamine B assays. Cells transfected with siRNA for $48 \mathrm{~h}$ were harvested for total-RNA and whole cell protein lysates purification and subsequent qRT-PCR and western blot analysis to evaluate knockdown efficiency and detect other relative signals.

Invasion assay. The invasion assay was performed using Matrigel Invasion Chambers with polyethylene terephthalate membrane on the bottom of the chambers $(8.0-\mu \mathrm{m}$ pore size) and Matrigel Basement Membrane Matrix from BD Biosciences (Bedford, MA) according to the manufacturer's protocol. Briefly, $5 \times 10^{4}$ cells in $0.5 \mathrm{ml}$ serum-free medium were seeded into each invasion chamber containing $27.2 \mu \mathrm{g}$ Matrigel Basement Membrane Matrix in $100 \mu 1$ in triplicate and incubated for $8 \mathrm{~h}$. Conditional medium harvested from cultured NIH3T3 cells was added to the bottom wells outside the chambers. The chambers were incubated at $37^{\circ} \mathrm{C}$ in the incubator containing $5 \% \mathrm{CO}_{2}$ for another $24 \mathrm{~h}$. Then the cells on the top side of the chamber were removed with a cotton swab, and the cells that invaded to the bottom side of the chamber were fixed and stained using HEMA3 stain set from Fisher Scientific, Co., LLC (Middletown, VA) following the manufacturer's instructions. The invasive cells on the lower surface of the membrane were pictured and cell numbers were counted under a microscope.

Sulforhodamine B assay. Cells after transfection with siRNA for $24 \mathrm{~h}$ were reseeded into 96 -well plates at 1,500 cells/well in $300 \mu \mathrm{l}$ media. Plates were fixed with $10 \%$ (w/v) trichloroacetic acid at $4^{\circ} \mathrm{C}$ each day for 5 days. Fixed cells were washed repeatedly with distilled water and dried. Then they were stained with $0.4 \%$ (w/v) sulforhodamine B for $30 \mathrm{~min}$ at room temperature, depleted, washed with $1 \%(\mathrm{v} / \mathrm{v})$ acetic acid repeatedly and dried. Finally, the dye was dissolved in $10 \mathrm{mmol} / \mathrm{l}$ Tris base solution for $5 \mathrm{~min}$ at room temperature with agitation. Absorbance was measured at $500 \mathrm{~nm}$ using $\mu$ Quant $^{\mathrm{TM}}$ Universal Microplate Spectrophotometer (BioTek Instruments, Inc.).

Western blot analysis. Whole-cell protein lysis buffer containing $1 \%$ Triton, $40 \mathrm{mmol} / 1 \mathrm{HEPES}, 120 \mathrm{mmol} / \mathrm{l} \mathrm{NaCl}, 1 \mathrm{mmol} / \mathrm{l}$ EDTA, $10 \mathrm{mmol} / 1$ pyrophosphate, $10 \mathrm{mmol} / 1$ glycerophosphate, $50 \mathrm{mmol} / 1 \mathrm{NaF}$ and $0.5 \mathrm{mmol} / 1 \mathrm{Na}_{3} \mathrm{VO}_{4}$, was stored at $4^{\circ} \mathrm{C}$. Protease inhibitors were added just before use. Cells were washed twice with cold PBS and incubated in lysis buffer on ice for $20 \mathrm{~min}$ and then centrifuged at $12,000 \mathrm{x}$ g at $4^{\circ} \mathrm{C}$ for $20 \mathrm{~min}$. Supernatant was quantified by Bradford assay and denatured in loading buffer. Protein lysates $(30-50 \mu \mathrm{g})$ were separated by $10 \%$ SDS-PAGE and then transferred to PVDF membranes using standard procedures. The membrane was incubated with the primary antibody overnight at $4^{\circ} \mathrm{C}$ and washed. After incubation in secondary antibody and wash, membranes were subjected to Supersignal west Pico chemiluminescent substrate from Thermo Scientific (Rockford, IL) and exposed.

Quantitative reverse transcription-polymerase chain reaction ( $q R T-P C R)$. Total-RNA from cells was extracted using TRIzol reagent from Invitrogen Life Technologies according to the manufacturer's protocol. TaqMan gene expression assays for miRNA were purchased from Applied Biosystems Inc. (Foster, CA) and used to quantify the relative expression of miR-9. U6 small nuclear RNA (U6 snRNA) was treated as a normalization control. For mRNA quantification, total-RNA was reverse transcribed following standard procedure using M-MLV reverse transcriptase (Promega, Madison, WI), and then subjected to quantitative PCR using Power SYBR-Green PCR Master mix from Applied Biosystems Inc. according to the manufacturer's instructions. Primers were described previously and synthesized by Invitrogen Life Technologies. Forward $(\mathrm{F})$ and reverse $(\mathrm{R})$ primer sequences are as follows: IRS-1, F, 5'-TGCTGGGGGTTTGGAGAATG-3' and R, 5'-GG CACTGTTTGAAGTCCTTGACC-3'; E-cadherin, F, 5'-CCCA CCACGTACAAGGGTC-3' and R, 5'-CTGGGGTATTGGGG GCATC-3'; Vimentin, F, 5'-ACCAGGTCCGTGTCCTCGT-3' and R, 5'-CTGCCCAGGCTGTAGGTG-3'; NFkB, F, 5'-CCTG AGACAAATGGGCTACAC-3' and R, 5'-TTTAGGGCTTTGG TTTACACGG-3'; snail, F, 5'-GCTGCAGGACTCTAATCCA GA-3' and R, 5'-ATCTCCGGAGGTGGGATG-3'; GAPDH, F, 5'-ATGGGGAAGGTGAAGGTCG-3' and R, 5'-GGGGTCAT TGATGGCAACAATA-3' (26-31). All real-time amplifications were measured in triplicates and performed with the ABI Prism 7300 sequence detection system from Applied Biosystems Inc. The fold-change of miR-9, IRS-1, E-cadherin and vimentin was calculated using the $2^{-\Delta \Delta \mathrm{Ct}}$ method.

RNA extraction from FFPE tissue samples. Total-RNA extracted from FFPE tissues was previously described (32). Briefly, the tumor sections (5- $\mu \mathrm{m}$ thick) were deparaffinized with xylene and washed three times with $100 \%$ ethanol. After drying, tissue pellets were added by $200 \mu \mathrm{l}$ of lysis buffer (20 mmol/1 Tris-HCl, pH 8.0, 20 mmol/1 EDTA, 2\% sodium dodecyl sulfate) and $10 \mu \mathrm{l}$ of proteinase $\mathrm{K}$ solution $(100 \mu \mathrm{g} / \mathrm{ml})$. After overnight incubation at $55^{\circ} \mathrm{C}, 1 \mathrm{ml}$ TRIzol reagent from Invitrogen Life Technologies was added to the sample and totalRNA was extracted according to the manufacturer's protocol.

Immunohistochemistry and scores. The slides were stained with IHC using the Ready-to-use EnVision ${ }^{\mathrm{TM}}+$ Dual Link System-HRP kit from Dako (Carpinteria, CA). The staining conditions were adjusted according to our laboratory experience. Briefly, each section was deparaffinized and rehydrated, and high-temperature antigen retrieval was achieved by heating the samples in $0.01 \mathrm{~mol} / 1$ citrate buffer in a domestic microwave oven at full power $(750 \mathrm{~W})$ for $15 \mathrm{~min}$, then the samples were immersed into methanol containing $0.3 \% \mathrm{H}_{2} \mathrm{O}_{2}$ for $30 \mathrm{~min}$ at $37^{\circ} \mathrm{C}$ to inactivate endogenous peroxidase. To eliminate nonspecific staining, we incubated the slides with appropriate preimmune serum for $30 \mathrm{~min}$ at room temperature, followed by incubation with primary antibody at $4^{\circ} \mathrm{C}$ overnight. Staining was done using the rabbit monoclonal antihuman IRS-1 from Epitomics, Inc. (Burlingame, CA) with a 


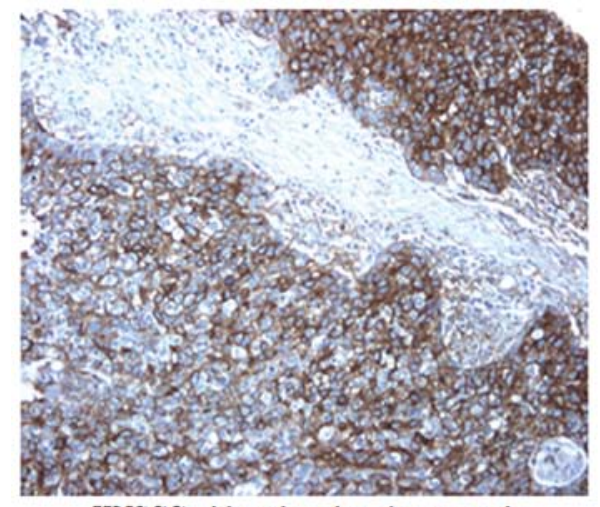

HNSCC without lymph node metastasis

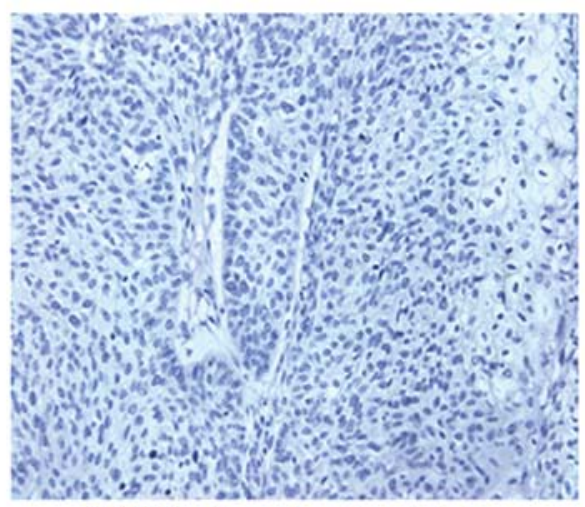

HNSCC with lymph nodemetastasis

Figure 1. IRS-1 protein expression in the specimens of HNSCC with or without lymph node metastasis by IHC. Left: strong positive staining. Right: negative staining. Representatives of IHC staining. Magnification, x200.

Table II. Analysis of the association between positive expression intensity of IRS-1 protein and lymph node metastasis of HNSCC.

\begin{tabular}{lcccc}
\hline & \multicolumn{4}{c}{ IRS-1 positive expression intensity } \\
\cline { 2 - 5 } Lymph node status & $+++(\%)$ & $++(\%)$ & $+(\%)$ & $-(\%)$ \\
\hline LNM (n=33) & $2(6.1)$ & $3(9.1)$ & $17(51.5)$ & $11(33.3)$ \\
No LNM (n=75) & $4(5.3)$ & $23(30.7)$ & $27(36.0)^{\mathrm{a}}$ & $21(28.0)$ \\
\hline
\end{tabular}

Chi-square test: ${ }^{\text {aStrong }}$ positive intensity $(+++$ and ++$)$ vs. weak positive intensity $(+), \mathrm{p}<0.05$.

1:100 dilution. Labeled polymer-HRP was added according to the manufacturer's instructions and incubated for $30 \mathrm{~min}$. Color reaction was developed using 3,3'-diaminobenzidine tetrachloride $(\mathrm{DAB})$ chromogen solution and all slides were counterstained with hematoxylin. Positive control slides were included in every experiment in addition to the internal positive controls. The specificity of the antibody was determined with matched IgG isotype antibody as a negative control. Moreover, a single band with correct molecular weight in western blot analysis was assured.

Immunohistochemical staining of sections were scored microscopically at $\mathrm{x} 400$ magnification in all available tumors by two pathologists using the qualitative scale described in the literature with minor modifications. In scoring the results, the intensity and the percentage of positive cells were taken into consideration. The number of cells stained was scored as 0 (no staining), $1+(<25 \%$ positive cells), $2+(>25$ and $<50 \%$ positive cells) and $3+(>50 \%$ positive cells). The intensity of staining was scored from $1+$ (weak) to $3+$ (strong). The immunoreactive score was categorized in three groups by comprehensive evaluation of the percentage of positive cells and staining intensity as reported previously. No staining was considered negative (0 score), weakly, moderately and strongly staining were considered positive (1,2 and 3 scores, respectively). Also, for statistical analysis, the staining results were classified into two groups depending on the intensity and the percentage of positive cells: 'negative group' (no staining, weak intensity and in $<10 \%$ positive cells); and the 'positive group' (strong intensity and in $>10 \%$ positive cells). Chi-square test was used for statistical analysis.

Statistical analysis. The statistical significance of differences between different groups was analyzed with two-sided unpaired Student's t-tests. Results were considered to be statistically significant at $\mathrm{p}<0.05$.

\section{Results}

IRS-1 expression correlates with clinicopathological parameters. To explore the relationship between IRS-1 expression and metastasis of HNSCC, we detected IRS- 1 expression levels in 108 HNSCC specimens using IHC methods. As shown in Table I, no correlation was found between the expression level of IRS- 1 and patient age and gender $(p>0.05)$. However, the positive expression rates of IRS-1 in well and moderately-differentiated carcinoma were higher than that in poorly-differentiated carcinoma. We also found there was a negative correlation between the IRS-1 expression and clinical staging $(\mathrm{p}<0.05)$. These results suggested that advanced clinical stages correlated with lower IRS-1 expression. Although we did not find any difference between the IRS-1 expression and lymph node metastasis, we found stronger positive expression of IRS-1 in tumors without lymph node metastasis rather than tumors with metastasis (Table II) when we further analyzed the positive expression intensity of IRS-1 protein. Fig. 1 shows representative staining of IRS-1 protein using IHC. These results suggest that decreased expression levels of IRS-1 are associated with lymph node metastasis of HNSCC.

IRS-1 expression decreases in the metastatic lymph node tumor tissues compared to primary tumor tissues of HNSCC patients. To further identify relationship of IRS-1 expression with metastasis, we compared IRS-1 mRNA expression in the primary and metastatic lymph node tumor tissues of HNSCC patients using qRT-PCR assay. As shown in Fig. 2, in 10 paired patient samples, 9 showed decreased expression of IRS-1 mRNA in lymph node tissue samples. The average decrease rate was $31 \%(\mathrm{p}<0.05)$. These results suggest that IRS-1 may serve as a suppressor of HNSCC metastasis. 


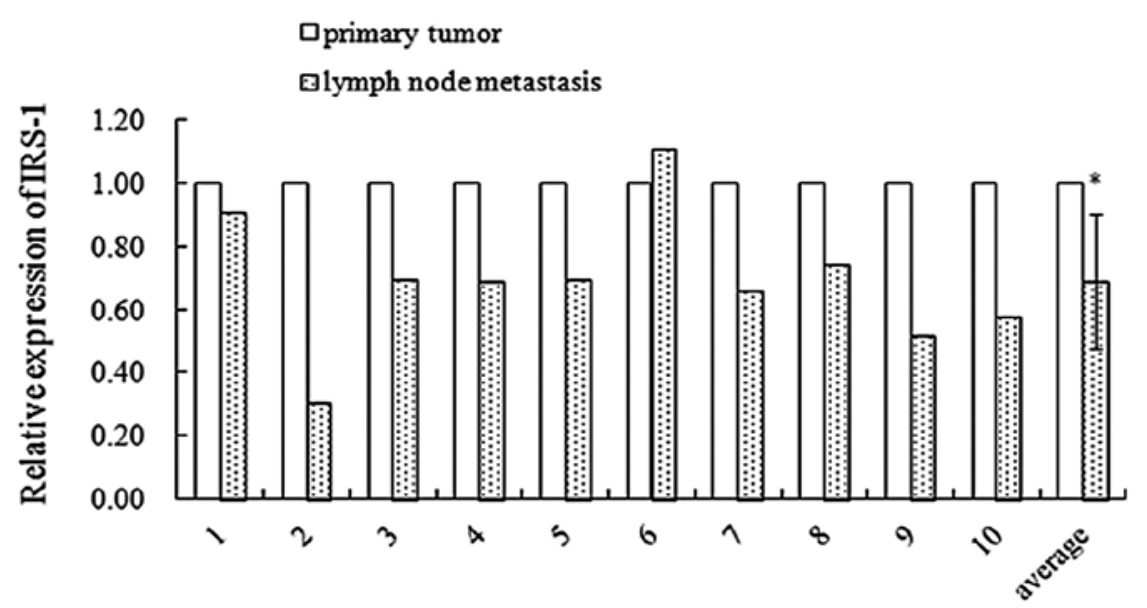

Figure 2. IRS-1 mRNA expression in paired lymph node and primary tumor tissues of 10 HNSCC patients. Total-RNA were extracted from FFPE tissue samples and subjected for qRT-PCR assay. The fold change of IRS-1 in lymph node was compared to primary tumor tissues of each patient and calculated using the $2^{-\Delta \Delta \mathrm{Ct}}$ method. Columns, $2^{-\Delta \Delta \mathrm{Ct}}$ values of each patient. Last column, a mean of ten patients; bars, SD. ${ }^{*} \mathrm{p}<0.05$.

A

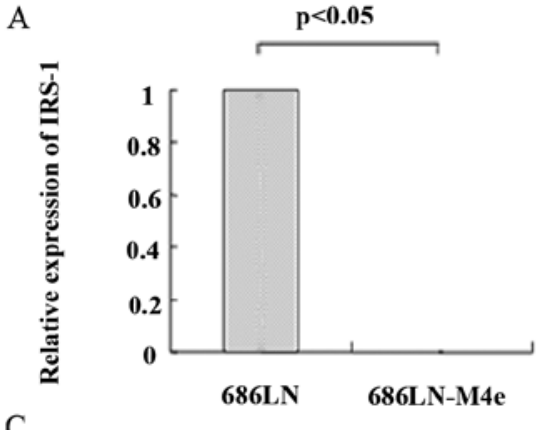

B
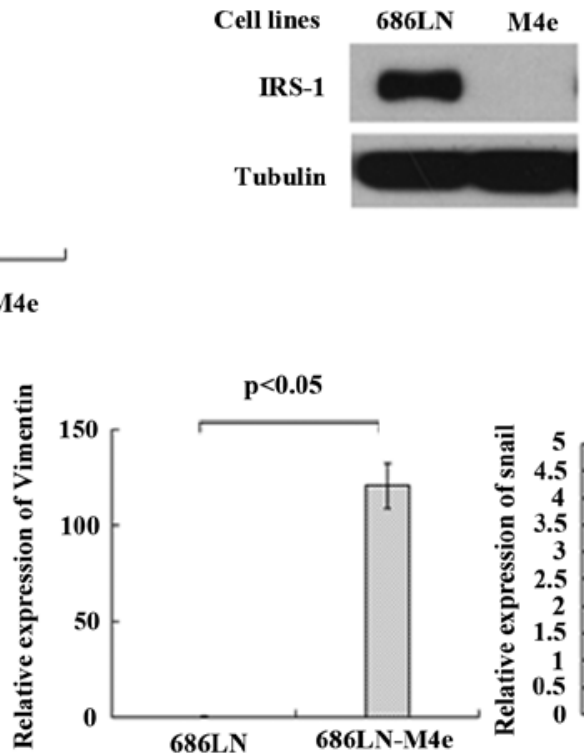

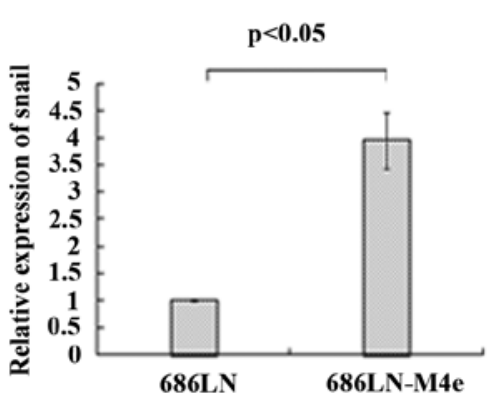

Figure 3. IRS-1 expression in paired highly and poorly metastatic HNSCC cell lines. (A) IRS-1 mRNA and (B) protein expression levels are lower in 686LNM4e cells compared with their parental 686LN cells. (A,C) Total-RNA was purified from 686LN and 686LN-M4e cells and then subjected to qRT-PCR assay. Columns, means of three replicate determinations; bars, SD. (B) Whole cell protein lysates were collected from cells aforementioned and subjected to western blot analysis. Representatives of three independent experiments.

IRS-1 expression is lower in highly metastatic HNSCC cells compared to expression in its parental poorly metastatic cells. To study the role of IRS-1 in HNSCC metastasis in vitro, we then detected IRS-1 expression levels in paired poorly-highly metastatic HNSCC cell lines. Highly metastatic 686LN-M4e cell lines were established from poorly metastatic 686LN cell lines through an in vivo selection mouse model as described previously (23,33). As shown in Fig. 3, both mRNA (Fig.3A) and protein levels (Fig. 3B) of IRS-1 were significantly decreased in highly metastatic $686 \mathrm{LN}-\mathrm{M} 4 \mathrm{e}$ cells when compared to poorly metastatic 686LN cells. Using a quantitative real-time PCR assay, the $\mathrm{Ct}$ value of IRS-1 products in $686 \mathrm{LN}-\mathrm{M} 4 \mathrm{e}$ cells was $28.54 \pm 0.10$, suggesting that $686 \mathrm{LN}-\mathrm{M} 4 \mathrm{e}$ cells had basal levels of IRS-1 mRNA expression. The $\Delta \mathrm{Ct}$ value (deduction of the $\mathrm{Ct}$ value of GAPDH) was $14.44 \pm 0.10$ in $686 \mathrm{LN}-\mathrm{M} 4 \mathrm{e}$ cells, while it was $5.03 \pm 0.19$ in $686 \mathrm{LN}$ cells, suggesting a much lower mRNA expression of IRS-1 in 686LN-M4e cells. A western blot analysis showed that protein levels of IRS-1 were undetectable in 686LN-M4e cells compared to 686LN cells. Thus, IRS-1 expression levels are decreased both at mRNA and protein levels in highly metastatic cell lines of HNSCC, suggesting IRS-1 to be a suppressor of head and neck cancer metastasis.

In addition, previous studies by Zhang et al (23) showed decreased E-cadherin and increased vimentin expression in 686LN-M4e cells compared with its parental 686LN cells, 


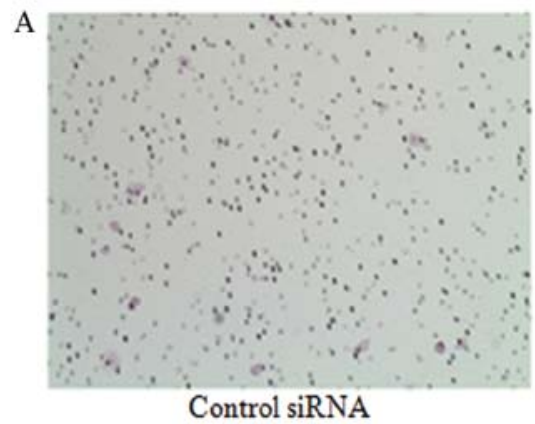

B

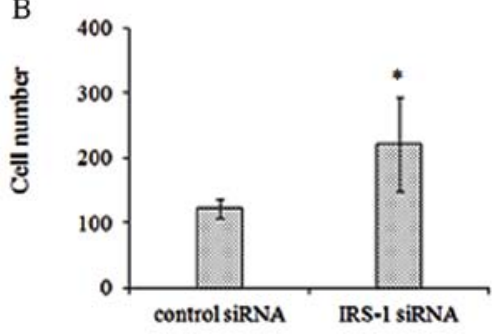

C

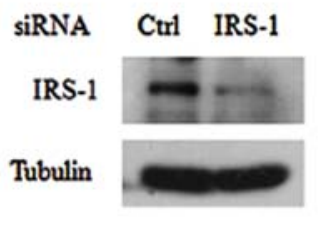

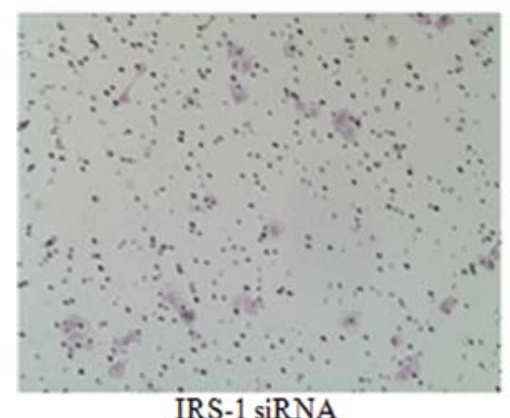

IRS-1 siRNA

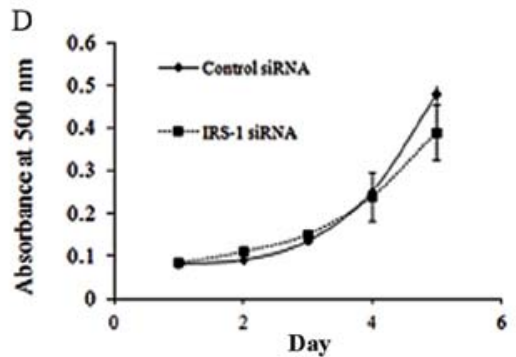

Figure 4. IRS-1 reduction promotes cell invasion. (A) Invasion assay. 686LN cells transfected with IRS-1 or control siRNA for $24 \mathrm{~h}$ were subjected to the matrigel invasion assay following product instructions. (B) Invaded cell number. Cells on the bottom side of the chamber were counted under a microscope. Columns, means of three replicate determinations; bars, SD. *p<0.05. (C) Knockdown efficacy of IRS-1 siRNA. Whole cell protein lysates were harvested from cells aforementioned and subjected to western blot analysis. Representatives of three independent experiments. Ctrl, control. (D) Growth curve of 686LN cells transfected with IRS-1 or control siRNA. 686LN cells were seeded to 6-well plates and transfected with IRS-1 or control siRNA for 24 h. Then, cells were reseeded to 96-well plates by 1,500 cells/well in $300 \mu 1$ media and subjected to SRB assay on different days as indicated. Points, means of three replicate determinations; bars, SD.

which suggested that highly metastatic cells gained EMT features. In this study, we detected E-cadherin and vimentin expression levels in these paired metastatic cell lines. As shown in Fig. 3C, E-cadherin mRNA expression was low and vimentin was high in 686LN-M4e cells, consistent with previous studies. We also detected increased expression of snail in 686LN-M4e cells, which is a well known transcriptional suppressor of E-cadherin. These findings further suggest EMT in promoting HNSCC metastasis.

Downregulation of IRS-1 expression promotes invasion of 686LN cells. Poorly metastatic 686LN cells have been previously shown to be less invasive than paired highly metastatic 686LN-M4e cells (24); therefore, to study the function of IRS-1 in HNSCC metastatsis, we first detected the impact of downregulating IRS-1 on invasive ability of 686LN cells (higher expression levels of IRS-1). As shown in Fig. 4A, increased cell numbers invading to the bottom side of the membrane after transfection with IRS-1 siRNA was observed as compared to control siRNA transfection. Quantification of cell numbers showed that the increase was statistically significant $(\mathrm{p}<0.05)$ (Fig. 4B). Western blot analysis confirmed the knockdown efficiency of IRS-1 as shown in Fig. 4C. These results suggest that downregulation of IRS-1 expression enhances the invasive potency of 686LN cells.

IRS-1 has been demonstrated to facilitate activation of a number of intracellular signaling molecules involved in cell growth, survival and transformation. Additionally, IRS-1 was reported to be correlated with cancer development and progression. Moreover, expression of IRS-1 or its active formtyrosine phosphorylated IRS-1 was shown to be elevated in certain types of tumor tissues compared to normal tissues $(5,9)$. Therefore, we performed a sulforhodamine B assay to evaluate the effect of IRS-1 on the growth of 686LN cells. Fig. 4D shows the growth curve of 686LN cells transfected with IRS-1 siRNA or control siRNA. Even though on Day 5, viability of cells with IRS-1 knockdown decreased slightly, the alteration is minor and not statistically significant $(\mathrm{p}>0.05)$. These results suggest that IRS-1 knockdown did not affect viability of 686LN cells.

Downregulation of IRS-1 expression induces epithelialmesenchymal transition. EMT is well-known to promote migration and invasion of many cancer cell types and plays crucial roles in cancer metastasis. E-cadherin downregulation has been considered as not only a marker of EMT, but also as a promoter of EMT. To further confirm the function of IRS-1 and clarify its mechanism, we detected critical markers of EMT. As shown in Fig. 5, downregulation of IRS-1 resulted in significant decrease of E-cadherin in both mRNA and protein levels and thus indicated EMT. But another EMT markervimentin did not increase as we had expected. Since previous findings have also shown distinctive E-cadherin expression in metastatic and nonmetastatic HNSCC human tissues (3), our findings suggest that knockdown of IRS-1 which induced loss of E-cadherin may account for enhanced cell invasive potency and EMT.

IRS-1 knockdown increases miR-9 expression in 686LN cells. It has been recently reported that miR-9 is the only miRNA that directly targets the 3' UTR of E-cadherin and induces its downregulation (20). Therefore, we further detected the effects of IRS-1 knockdown on miR-9 expression in 686LN cells. Our results showed that miR-9 increased 5.15-fold (Fig. 6A) after IRS-1 siRNA transfection for $48 \mathrm{~h}$ as compared with control siRNA, and the parallel expression of E-cadherin decreased 

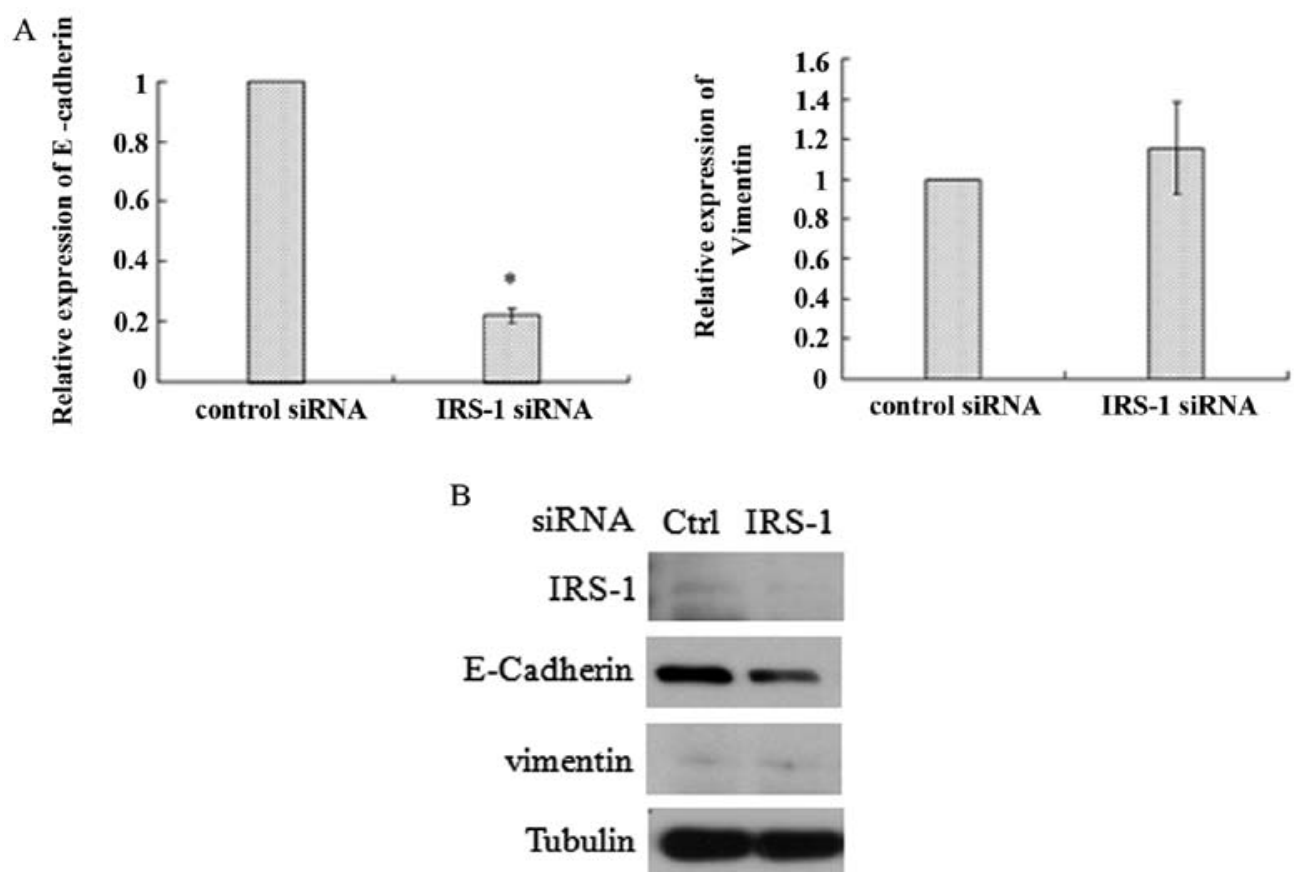

Figure 5. Markers of EMT in 686LN cells transfected with control or IRS-1 siRNA. (A) QRT-PCR assay of E-cadherin and vimentin in 686LN cells after transfection with control or IRS-1 siRNA. Columns, means of three replicate determinations; bars, SD. (B) Whole cell lysates were collected and subjected to western blot analysis. A representative of three independent experiments. Ctrl, control.

A

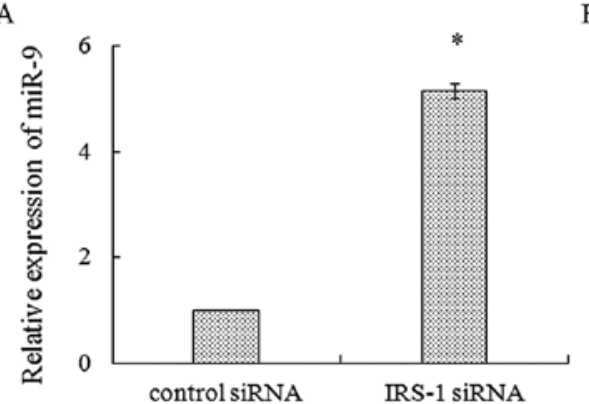

$\mathrm{C}$

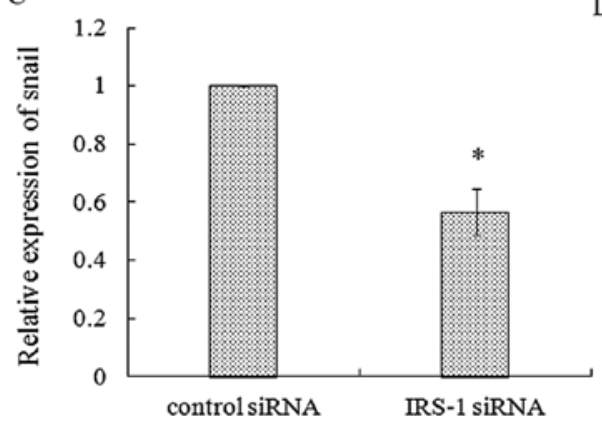

B

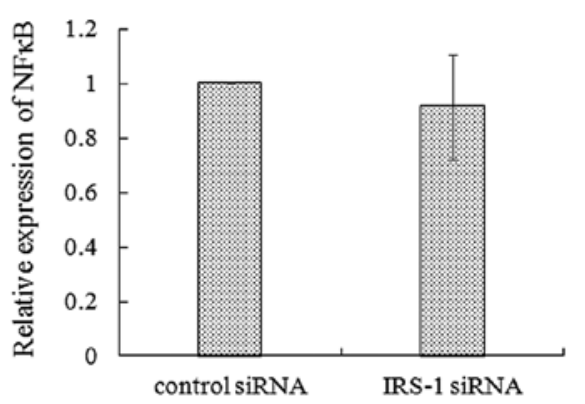

D

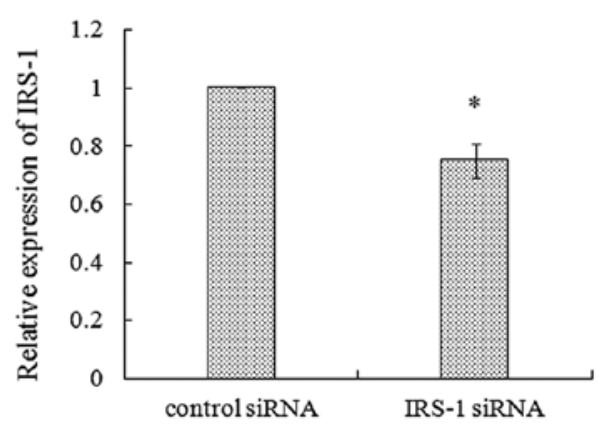

Figure 6. Knockdown of IRS-1 mediated miR-9 and its target genes expression in 686LN cells. 686LN cells transfected with control or IRS-1 siRNA for $48 \mathrm{~h}$ were subjected to qRT-PCR assay. The fold-change of (A) miR-9, (B) NFKB, (C) snail and (D) IRS-1 was calculated using the $2^{-\Delta \Delta C t}$ method. Columns, means of three replicate determinations; bars, SD. "p $<0.05$.

significantly (Fig. 5A). NFkB has been reported as another target gene of miR-9 in gastric cancer (34). NFkB has been demonstrated to play important roles in cell growth, survival, apoptosis and motility, thus promoting cancer progression, malignancy and metastasis (35). Therefore, we also evaluated NFKB expression after IRS-1 siRNA knockdown. As shown in Fig. 6B, no alteration of $\mathrm{NF} \kappa \mathrm{B}$ was observed. In addition, we found decreased expression of snail in 686LN cells with IRS-1 knockdown, suggesting snail may not be involved in E-cadherin downregulation (Fig. 6C). Fig. 6D shows significant decrease of IRS-1 after siRNA transfection in the experiments aforementioned. These results suggested the important role of miR-9 mediated downregulation of E-cadherin in IRS-1 knockdown-induced invasion of 686LN cells. 


\section{Discussion}

Metastasis is responsible for the high morbidity of head and neck cancer patients. Thus, accurate diagnosis and effective novel therapeutic regimens of metastasis are pivotal for treatment of HNSCC. In this study, we found IRS-1 expression to be decreased in the metastatic lymph node tumor tissues of HNSCC patients compared with the primary tumor tissues. Additionally, we identified decreased expression of IRS-1 in highly metastatic HNSCC cells (686LN-M4e) compared with poorly metastatic cells $(686 \mathrm{LN})$. These results indicate that IRS-1 downregulation may be a predictor for metastasis of HNSCC. In the other cohort analyzed by IHC, even though the positive staining rate for IRS-1 protein had no difference between HNSCCs with and without lymph node metastasis, we found decreased positive staining intensity of IRS-1 protein in HNSCCs with lymph node metastasis. These results further suggest that the low expression of IRS-1 may predict metastasis of HNSCC. Barnes et al (36) have shown increased expression of insulin-like growth factor type I receptor (IGF-IR) both in cell lines and tumors of head and neck cancer. Even though IRS-1 is the major mediator of IGF-IR activation, its expression levels and activity have not been detected and are unknown in head and neck cancer. In the present study, we detected the IRS-1 expression in 108 HNSCCs using IHC and found that IRS-1 expression was negatively correlated with histological grade and clinical stages. These results suggest IRS-1 may be developed as a new predictive marker for progression and metastasis of HNSCC. This may help clinicians to predict the biological behavior of tumors, specifically in small biopsy specimens, and may provide more accurate staging systems and means of monitoring patients with this type of cancer (37).

IRS-1, as a positive effector of growth factors and hormones, has been shown to facilitate the activation of a number of intracellular signaling molecules, especially PI3K and MAPK, involved in cell metabolism, growth, survival, transformation and motility, thus IRS-1 is considered tumorigenic. Studies have also demonstrated that IRS-1 can be both tumor promoter and suppressor (5). Currently, studies involving the role of IRS-1 in tumor transformation and metastasis are controversial. In breast cancer, prostate cancer and neuroblastoma, IRS-1 has been confirmed to induce cell motility and transformation, suggesting it as a positive regulator $(4,5)$. But in other studies in breast cancer, Ma et al (10) and Gibson et al (38) have demonstrated that suppression of IRS-1 promotes mammary tumor metastasis using an IRS-1 knockout mouse model. In lung cancer, Shi et al (11) showed that IRS-1 suppresses cell migration, invasion and EMT. In this study, for the first time, we showed that in HNSCC cells, downregulation of IRS-1 enhances invasive potency and induces EMT. Recent findings by Houghton et al (7) demonstrated that silencing IRS-1 expression increases proliferation of A549 cells, suggesting it as a tumor suppressor in lung cancer. This is consistent with studies by Shi et al (11) that IRS-1 is a metastatic suppressor. Therefore, the IRS-1 impact may depend on cancer types.

When cells undergo EMT, they lose the features of epithelial cells and acquire features of mesenchymal cells. Therefore, hallmarks of epithelial cells such as E-cadherin decrease and hallmarks of mesenchymal cells including vimentin and fibronection increase. Among them, loss of E-cadherin expression is the critical step of EMT in tumor metastasis. Other markers of EMT also include elevated transcriptional factors such as snail, slug, twist, bmi1 and SIP1 that suppress E-cadherin expression (12-14). In this study, we detected a significant decrease of E-cadherin after IRS-1 knockdown, but we did not observe an increase in vimentin expression. EMT is a multi-step and complicated process; therefore, the alterations of markers are sequential events. It is possible that elevation of vimentin may be present in the later stages of IRS-1 induced EMT, since 686LN-M4e cells have extremely higher levels of vimentin as compared with their parental $686 \mathrm{LN}$ cells (Fig. 4A). We could not rule out other possibilities, such as other markers than vimentin that may play essential roles for IRS-1 induced EMT of head and neck cancer cells. Since loss of E-cadherin is not only the most common indicator of EMT, but also a strong promoter of EMT, our results suggest that IRS-1 may suppress EMT.

In recent years, miRNA has emerged as a new class of gene expression regulators that are predicted to be involved in $30 \%$ of human gene regulation. Deregulation of miRNAs has been confirmed in many kinds of disease including cancer. Moreover, different deregulated miRNA expression profiles may serve as predictive markers for cancer types and subtypes, drug sensitivity and different therapeutic strategies $(16,17,19)$. Overexpressed miRNAs in tumors function through downregulating oncogene expression, while decreased miRNAs in tumors contribute through upregulating expression of tumor suppressors. MiR-9 has been suggested as both an oncogene and a tumor suppressor, depending on different cancer types. MiR-9 acts as a putative tumor suppressor gene in recurrent ovarian cancer, gastric cancer and medulloblastoma. Its predicted target genes include RAB34, TrkC, CDX2 and FOXO1 $(19,39,40)$. Wan et al (34) demonstrated that miR-9 suppresses gastric cancer through direct targeting of $\mathrm{NF} K \mathrm{~B}$. Since NFאB plays important roles in tumor metastasis by increasing VEGF and MMPs transcription, we also investigated its expression (35). Our results showed that NFkB was not affected by IRS-1 knockdown. In addition, upregulation of miR-9 would result in downregulation of NFkB expression and its downstream signals in metastasis; therefore, IRS-1induced upregulation of miR-9 does not seem likely to function through NFאB. We also found downregulation of snail by IRS-1 knockdown, which suggested that decreased E-cadherin expression may not be through snail-mediated transcriptional regulation. Recently, miR-9 has been confirmed to directly target E-cadherin in breast cancer, and thus promotes EMT and tumor metastasis (20). In this study, we identified upregulation of miR-9 and correlated downregulation of E-cadherin after IRS-1 knockdown; thus, our results suggest that miR-9 mediated suppression of E-cadherin is involved in the IRS-1 suppressive impact on tumor metastasis.

In summary, we identified negative correlation of IRS-1 expression with tumor metastasis both in tissue samples and cell lines. Furthermore, we showed that downregulation of IRS-1 expression by siRNA promotes poorly metastatic HNSCC cells invasive potency and induces EMT possibly through upregulation of miR-9 expression and subsequent loss of E-cadherin. Our findings indicate that IRS-1 may have a negative impact on the survival of HNSCC patients and may serve as a new potential target for cancer therapy. 


\section{Acknowledgements}

This study was supported by grants from the National Natural Science Foundation of China (nos. 30873099, 81172004 and 81102458). We thank Dr Zhuo Chen for providing 686LN and 686LN-M4e cell lines (Georgia, USA). We thank Dr Heath Elrod for editing the article.

\section{References}

1. Jemal A, Siegel R, Xu J and Ward E: Cancer statistics, 2010. CA Cancer J Clin 60: 277-300, 2010.

2. Som PM: Detection of metastasis in cervical lymph nodes: CT and MR criteria and differential diagnosis. AJR Am J Roentgenol 158: 961-969, 1992.

3. Muller S, Su L, Tighiouart M, et al: Distinctive E-cadherin and epidermal growth factor receptor expression in metastatic and nonmetastatic head and neck squamous cell carcinoma: predictive and prognostic correlation. Cancer 113: 97-107, 2008.

4. Dearth RK, Cui X, Kim HJ, Hadsell DL and Lee AV: Oncogenic transformation by the signaling adaptor proteins insulin receptor substrate (IRS)-1 and IRS-2. Cell Cycle 6: 705-713, 2007.

5. Metz HE and Houghton AM: Insulin receptor substrate regulation of phosphoinositide 3-kinase. Clin Cancer Res 17: 206-211, 2011.

6. Chang Q, Li Y, White MF, Fletcher JA and Xiao S: Constitutive activation of insulin receptor substrate 1 is a frequent event in human tumors: therapeutic implications. Cancer Res 62: 6035$6038,2002$.

7. Houghton AM, Rzymkiewicz DM, Ji H, et al: Neutrophil elastase-mediated degradation of IRS-1 accelerates lung tumor growth. Nat Med 16: 219-223, 2010.

8. Koda M, Sulkowska M, Kanczuga-Koda L and Sulkowski S: Expression of insulin receptor substrate 1 in primary breast cancer and lymph node metastases. J Clin Pathol 58: 645-649, 2005.

9. Dearth RK, Cui X, Kim HJ, et al: Mammary tumorigenesis and metastasis caused by overexpression of insulin receptor substrate 1 (IRS-1) or IRS-2. Mol Cell Biol 26: 9302-9314, 2006.

10. Ma Z, Gibson SL, Byrne MA, Zhang J, White MF and Shaw LM: Suppression of insulin receptor substrate 1 (IRS-1) promotes mammary tumor metastasis. Mol Cell Biol 26: 9338-9351, 2006.

11. Shi J, Wang DM, Wang CM, et al: Insulin receptor substrate-1 suppresses transforming growth factor-beta1-mediated epithelial-mesenchymal transition. Cancer Res 69: 7180-7187, 2009.

12. Vernon AE and LaBonne C: Tumor metastasis: a new twist on epithelial-mesenchymal transitions. Curr Biol 14: R719-R721, 2004.

13. Martin A and Cano A: Tumorigenesis: Twist1 links EMT to selfrenewal. Nat Cell Biol 12: 924-925, 2010.

14. McCarthy N: Metastasis: Twisting BMI1. Nat Rev Cancer 10: $666,2010$.

15. Peinado H, Olmeda D and Cano A: Snail, Zeb and bHLH factors in tumour progression: an alliance against the epithelial phenotype? Nat Rev Cancer 7: 415-428, 2007.

16. Eulalio A, Huntzinger E and Izaurralde E: Getting to the root of miRNA-mediated gene silencing. Cell 132: 9-14, 2008.

17. Ventura A and Jacks T: MicroRNAs and cancer: short RNAs go a long way. Cell 136: 586-591, 2009.

18. Croce CM and Calin GA: miRNAs, cancer, and stem cell division. Cell 122: 6-7, 2005

19. Nicoloso MS, Spizzo R, Shimizu M, Rossi S and Calin GA: MicroRNAs--the micro steering wheel of tumour metastases. Nat Rev Cancer 9: 293-302, 2009.

20. Ma L, Young J,Prabhala H, et al: miR-9, a MYC/MYCN-activated microRNA, regulates E-cadherin and cancer metastasis. Nat Cell Biol 12: 247-256, 2010
21. Burk U, Schubert J, Wellner U, et al: A reciprocal repression between ZEB1 and members of the miR-200 family promotes EMT and invasion in cancer cells. EMBO Rep 9: 582-589, 2008.

22. Wellner U, Schubert J, Burk UC, et al: The EMT-activator ZEB1 promotes tumorigenicity by repressing stemness-inhibiting microRNAs. Nat Cell Biol 11: 1487-1495, 2009.

23. Zhang X, Su L, Pirani AA, et al: Understanding metastatic SCCHN cells from unique genotypes to phenotypes with the aid of an animal model and DNA microarray analysis. Clin Exp Metastasis 23: 209-222, 2006.

24. Zhang X, Liu Y, Gilcrease MZ, et al: A lymph node metastatic mouse model reveals alterations of metastasis-related gene expression in metastatic human oral carcinoma sublines selected from a poorly metastatic parental cell line. Cancer 95: 1663-1672, 2002.

25. Zhang H, Su L, Muller S, et al: Restoration of caveolin-1 expression suppresses growth and metastasis of head and neck squamous cell carcinoma. Br J Cancer 99: 1684-1694, 2008.

26. Cantarini MC, de la Monte SM,Pang M, et al: Aspartyl-asparagyl beta hydroxylase over-expression in human hepatoma is linked to activation of insulin-like growth factor and notch signaling mechanisms. Hepatology 44: 446-457, 2006.

27. Gregory PA, Bert AG, Paterson EL, et al: The miR-200 family and miR-205 regulate epithelial to mesenchymal transition by targeting ZEB1 and SIP1. Nat Cell Biol 10: 593-601, 2008.

28. Bazzoni F, Rossato M, Fabbri M, et al: Induction and regulatory function of miR-9 in human monocytes and neutrophils exposed to proinflammatory signals. Proc Natl Acad Sci USA 106: 5282-5287, 2009.

29. Dubrovska A, Kim S, Salamone RJ, et al: The role of PTEN/ $\mathrm{Akt} / \mathrm{PI} 3 \mathrm{~K}$ signaling in the maintenance and viability of prostate cancer stem-like cell populations. Proc Natl Acad Sci USA 106: 268-273, 2009.

30. Tiede S, Kloepper JE, Ernst N, Poeggeler B, Kruse C and Paus R: Nestin in human skin: exclusive expression in intramesenchymal skin compartments and regulation by leptin. J Invest Dermatol 129: 2711-2720, 2009.

31. Shao M, Cao L, Shen C, et al: Epithelial-to-mesenchymal transition and ovarian tumor progression induced by tissue transglutaminase. Cancer Res 69: 9192-9201, 2009.

32. Tsuji S, Hisaoka M, Morimitsu Y, et al: Detection of SYT-SSX fusion transcripts in synovial sarcoma by reverse transcriptionpolymerase chain reaction using archival paraffin-embedded tissues. Am J Pathol 153: 1807-1812, 1998.

33. Chen Z, Zhang K, Zhang X, et al: Comparison of gene expression between metastatic derivatives and their poorly metastatic parental cells implicates crucial tumor-environment interaction in metastasis of head and neck squamous cell carcinoma. Clin Exp Metastasis 20: 335-342, 2003.

34. Wan HY, Guo LM, Liu T, Liu M, Li X and Tang H: Regulation of the transcription factor NF-kappaB1 by microRNA-9 in human gastric adenocarcinoma. Mol Cancer 9: 16, 2010.

35. Garg A and Aggarwal BB: Nuclear transcription factor-kappaB as a target for cancer drug development. Leukemia 16: 1053-1068, 2002.

36. Barnes CJ, Ohshiro K, Rayala SK, El-Naggar AK and Kumar R: Insulin-like growth factor receptor as a therapeutic target in head and neck cancer. Clin Cancer Res 13: 4291-4299, 2007.

37. Forastiere A, Koch W, Trotti A and Sidransky D: Head and neck cancer. N Engl J Med 345: 1890-1900, 2001.

38. Gibson SL, Ma Z and Shaw LM: Divergent roles for IRS-1 and IRS-2 in breast cancer metastasis. Cell Cycle 6: 631-637, 2007.

39. Myatt SS, Wang J, Monteiro LJ, et al: Definition of microRNAs that repress expression of the tumor suppressor gene FOXO1 in endometrial cancer. Cancer Res 70: 367-377, 2010.

40. Rotkrua P, Akiyama Y, Hashimoto Y, Otsubo T and Yuasa Y: MiR-9 downregulates CDX2 expression in gastric cancer cells. Int J Cancer 129: 2611-2620, 2011. 